\title{
Control System Design subject to SNR Constraints *
}

\author{
Eduardo I. Silva ${ }^{\text {a }}$, Graham C. Goodwin ${ }^{b}$, Daniel E. Quevedo ${ }^{\text {b }}$ \\ ${ }^{a}$ Departamento de Electrónica, Universidad Técnica Federico Santa María, Valparaíso, Chile \\ ${ }^{\mathrm{b}}$ School of Electrical Engineering and Computer Science, The University of Newcastle, Australia
}

\begin{abstract}
This paper deals with networked control systems comprising LTI plants controlled over scalar additive noise channels subject to signal-to-noise ratio (SNR) constraints. We present a general framework, based upon convex optimization concepts, that can accommodate several situations of interest. Our results make explicit the fact that feedback around the channel plays a key role in reducing the minimal SNR that is compatible with stability. The results also provide a characterization of the best achievable performance subject to an SNR constraint. We apply the results to specific networked control architectures, and provide a numerical example.
\end{abstract}

Key words: Networked control systems, Signal-to-noise ratio, Linear time-invariant systems, Optimal control, Networks

\section{Introduction}

Networked control systems (NCSs) are control systems that operate subject to communication constraints [1]. Such constraints arise from the characteristics of practical channels. For example, the use of digital networks usually implies the presence of data-rate limits, random delays, or data dropouts (see, e.g., papers in [1]). On the other hand, analog communication systems are usually subject to power (or variance) constraints, or signal-tonoise ratio (SNR) constraints [15]. In this paper, we consider a simple setting where the communication systems are assumed analog and SNR constraints are present. Our work extends the results in [5], where the study of SNR constrained NCSs was initiated. An overview of prior work is given below.

The authors of [5] study an NCS where an LTI SISO plant is controlled over an additive white Gaussian noise channel with input power constraint $V$ and channel noise variance $\sigma^{2}$. It is assumed in [5] that signals are sent over the channel without any pre- or post-processing (i.e., no coding is employed), and that the channel noise is the only exogenous signal in the feedback loop. For the

\footnotetext{
* This paper was not presented at any IFAC meeting. Corresponding author: E.I. Silva, Departamento de Electrónica, Universidad Técnica Federico Santa María, Casilla 100-V, Valparaíso, Chile. Tel. +56-32-2654495

Email address: eduardo.silva@usm.cl (Eduardo I. Silva).
}

discrete time static state feedback case, [5] shows that a necessary and sufficient condition for the existence of a controller that guarantees the stability of the feedback loop, and achieves an SNR less than or equal to the maximal admissible SNR, namely $\Gamma \triangleq V / \sigma^{2}$, is given by

$$
\Gamma>\left(\prod_{i=1}^{n_{p}}\left|p_{i}\right|^{2}\right)-1,
$$

where $p_{i}$ is the $i^{\text {th }}$ unstable plant pole. When dynamic LTI output feedback is employed, then the requirements on $\Gamma$ are more demanding than those in (1), except when the plant is minimum-phase (MP) and of relative degree one [5]. The work [14] extends [5] to the noisy plant case and shows that, if general (possibly time-varying and nonlinear) coders and controllers are considered, then (1) remains necessary for the mean square stability of the loop when state feedback is used. The results in [14] also give a lower bound on the mean square norm of the plant state. This bound shows that, due to the presence of process disturbances, the loop performance becomes arbitrarily poor if the SNR approaches its minimal admissible value. This result is intuitive and consistent with related results in [20]. It is not clear, however, whether or not the performance bounds given in [14] are tight. For the case of noiseless MP plant models with relative degree one, [14] also showed that condition (1) is sufficient for the existence of a stabilizing LTI output feedback law which satisfies the SNR constraint. Another relevant work is [10], where the undesirable robustness and per- 
formance related properties of the designs discussed so far are overcome by incorporating an additional penalizing term in the problem formulation. Related results are presented in $[5,22]$.

Performance related issues are explored in [23,24], where the form of the optimal (with respect to stabilization) sensitivity function is studied, and the gap between the SNR achieved by a given sensitivity function and the minimal SNR for stability, is used as a means to measure the SNR needed to give performance guarantees.

A difficulty associated with the results in $[23,24]$ is that they do not address the question of what is the best sensitivity function (in some sense) for a given SNR constraint. Also, they do not address the problem of optimal design with SNR constraints. Partial solutions to the latter problem have been proposed in $[16,21,30]$, where a nominal controller design is embellished with an optimally designed coding system aimed at reducing the stationary tracking error variance. The more fundamental question of controller design has been explored in [12] In that work, one degree of freedom control schemes for noisy discrete-time LTI plants are studied assuming an additive white Gaussian noise feedback channel with pre- and post scaling factors. The results in [12] characterize the best achievable performance (as measured by the stationary plant output variance), and the associated optimal LTI controllers and scaling factors, for MP plants that have relative degree one. A particular feature of the setup of [12] is that the channel input power constraint is, essentially, a constraint on the plant output variance which, in turn, is the signal used to measure performance [12, p. 3960]. This feature is not always present in NCS problems subject to SNR constraints (see [32]).

Recent work documented in $[11,13]$ uses a setup similar to that of [12] and studies minimization of the plant output variance at a given terminal time by means of general (possibly time-varying and nonlinear) control architectures. For MP plants having relative degree one, it is shown in $[11,13]$ that the best achievable performance can be achieved by linear time-varying strategies. These results are important. However, minimizing the output variance at a given instant may yield closed loops with poor transient performance [13].

The present paper makes two contributions. The first is a general framework for studying the interplay between SNR constraints and the stability and stationary performance of LTI feedback loops that include a scalar additive noise communication channel. As a second contribution, we show that it is possible to achieve, for arbitrary plant models and arbitrary noise sources, MSS at SNRs arbitrarily close to the bound (1) using LTI output feedback schemes, provided feedback around the channel is available. The paper extends our previous work described in $[16,32]$ to include general LTI control architectures, and to cover the case of channels with feedback.
The remainder of the paper is organized as follows: Section 2 presents notation. Section 3 discusses the channel model adopted in this paper. Section 4 presents assumptions and states the problem of interest. Sections 5 and 6 study stability and performance related questions in a general feedback architecture. Section 7 discusses specific architectures, and an example is provided in Section 8. Conclusions are given in Section 9.

\section{Notation}

$\mathbb{N}_{0} \triangleq\{0,1, \cdots\}, \mathbb{R}^{+} \triangleq\{x \in \mathbb{R}: 0<x<\infty\}$, $\mathbb{R}_{0}^{+} \triangleq \mathbb{R}^{+} \cup\{0\} ; \operatorname{vec}\{\cdot\}$ denotes the column stacking operator, $\mathrm{vec}^{-1}\{\cdot\}$ its inverse, and $\otimes$ the Kronecker product [3]. Given any scalar $x,|x|$ denotes its magnitude and $\bar{x}$ its complex conjugate; $(\cdot)^{T}$ denotes transpose and $(\cdot)^{H}$ conjugate transpose. We use $z$ as both the argument of the z-transform and as the forward shift operator, where the meaning is clear from the context.

$\mathcal{R}$ is the set of all real rational discrete-time (possibly multivariate) transfer functions. We define the following subsets of $\mathcal{R}: \mathcal{R}_{p}$ contains all proper transfer functions, $\mathcal{R}_{s p}$ contains all strictly proper transfer functions, $\mathcal{R} \mathcal{H}_{\infty}$ (resp. $\overline{\mathcal{R H}}_{\infty}$ ) contains all the stable (resp. marginally stable) and proper transfer functions, $\mathcal{U}_{\infty}$ (resp. $\overline{\mathcal{U}}_{\infty}$ ) contains all square transfer functions in $\mathcal{R} \mathcal{H}_{\infty}$ with inverses in $\mathcal{R} \mathcal{H}_{\infty}$ (resp. in $\left.\overline{\mathcal{R H}}_{\infty}\right), \mathcal{R H}_{2}$ contains all stable and strictly proper transfer functions, and $\mathcal{R H}_{2}^{\perp}$ contains all transfer functions that have no poles in $\{z \in \mathbb{C}$ : $|z| \leq 1\}$. Any $A(z) \in \mathcal{R}$ with no poles on the unit circle belongs to $\mathcal{L}_{2}$. For such $A(z)$ we define the 2 -norm as usual, and denote it by $\|\cdot\|_{2}$ (see, e.g., [35]). Any $A(z) \in \mathcal{R}$ with no poles on the unit circle can be written as $A(z)=[A(z)]_{\mathcal{H}_{2}^{\perp}}+[A(z)]_{\mathcal{H}_{2}}$, where $[A(z)]_{\mathcal{H}_{2}^{\perp}} \in \mathcal{R H}_{2}^{\perp}$ and $[A(z)]_{\mathcal{H}_{2}} \in \mathcal{R H}_{2}[35]$. When no confusion arises, we omit the argument $z$ in $A(z)$. We write $\left.\{A(z)\}\right|_{z=0}$ instead of $A(0)$, and use $A(z)^{\sim}$ as shorthand for $A\left(z^{-1}\right)^{T}$.

A random process $\{x(k)\}_{k \in \mathbb{N}_{0}}$ is usually referred to as $x$. The variance, at time instant $k$, of a process $x$ is denoted via $\sigma_{x}^{2}(k)$. Similarly, if $x$ is a random variable, then $\sigma_{x}^{2}$ denotes its variance. We define (if it exists) the stationary variance of $x$ via $\sigma_{x}^{2} \triangleq \lim _{k \rightarrow \infty} \sigma_{x}^{2}(k)$. If $x$ is a wide sense stationary (wss) (asymptotically wss) process, then $S_{x}\left(e^{j \omega}\right)$ denotes its (stationary) power spectral density (PSD) and $\Omega_{x}(z)$ denotes any spectral factor of $S_{x}\left(e^{j \omega}\right)$, i.e., $\Omega_{x}\left(e^{j \omega}\right) \Omega_{x}\left(e^{j \omega}\right)^{H} \triangleq S_{x}\left(e^{j \omega}\right)$. A random variable (process) is a second order one if and only if it has finite mean and finite second order moments for all time for all time instants $k \in \mathbb{N}_{0}$, and also when $k \rightarrow \infty$. 


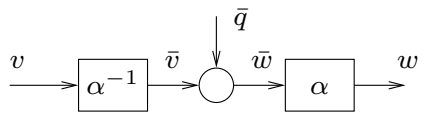

(a)

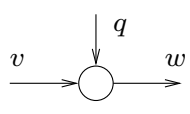

(b)
Fig. 1. (a) AWN channel with pre- and post-scaling factors, and (b) equivalent rewriting.

\section{Additive White Noise Channels with SNR Constraints}

This section motivates the channel model adopted in this paper. Consider a standard scalar additive white noise (AWN) channel with a (stationary) input variance constraint $[5,8,15]$. In such a channel, the input $\bar{v}$ and output $\bar{w}$ are related via $\bar{w}=\bar{v}+\bar{q}, \bar{q}$ is a zero mean white noise sequence with variance $\sigma_{\bar{q}}^{2} \in \mathbb{R}^{+}$, and there exists an upper bound on the stationary variance of the input $\bar{v}$ (assuming such stationary variance to exist), i.e., there exists $V \in \mathbb{R}^{+}$such that

$$
\sigma_{\bar{v}}^{2} \leq V
$$

The situation described above assumes that both $V$ and $\sigma_{\bar{q}}^{2}$ are parameters of a physical situation and, thus, they are fixed and given. AWN channels are often used to model wireless links [15, Chapter 4], and stand as cornerstones of communication theory [8].

In practice, the signal $\bar{v}$ corresponds to a scaled version of the signal of interest. It is thus both theoretically and practically sound to use an AWN channel with both preand post-scaling factors, as depicted in Figure 1(a). In that figure, $\alpha \in \mathbb{R}^{+}$is a parameter to be chosen by the designer (see also [12]). It is immediate to see that the signals $v$ and $w$ in Figure 1(a) are related via $w=$ $v+q$, where the equivalent noise $q \triangleq \alpha \bar{q}$ is a zero mean white noise sequence with a variance $\sigma_{q}^{2}=\left(\alpha \sigma_{\bar{q}}\right)^{2}$ (see Figure $1(\mathrm{~b}))$. Since $\alpha$ is a designer's choice, $\sigma_{q}^{2}$ becomes a design parameter in $\mathbb{R}^{+}$. With the previous definitions, the variance constraint in (2) can be written as

$$
\gamma \triangleq \frac{\sigma_{v}^{2}}{\sigma_{q}^{2}} \leq \frac{V}{\sigma_{\bar{q}}^{2}}
$$

Thus, the use of scaling factors around AWN channels turns variance constraints on $\bar{v}$ into an upper bound on the ratio between the variance of the signal $v$ and that of the equivalent noise source $q$. For a fixed power constraint $V$ and a fixed underlying noise source $\bar{q}$, the bound on $\gamma$ is also fixed and corresponds to the maximal available channel SNR.

Motivated by the previous discussion we introduce the following definition:

Definition 1 A scalar SNR constrained AWN channel is a device with scalar input $v$ and scalar output $w$ related via $w=v+q$, where $q$ is a zero mean white noise sequence with a variance that is a decision variable in $\mathbb{R}^{+}$, and $\gamma$ defined in (3) satisfies $\gamma \leq \Gamma$ for some given $\Gamma \in \mathbb{R}^{+}$.

Remark 2 Definition 1 implicitly assumes that $v$ has a stationary variance. This holds provided that the channel is part of an internally stable LTI system and that all exogenous signals are wss.

Remark 3 We will use in the paper "channel" as a synonym for "SNR constrained AWN channel".

According to the motivation leading to Definition 1, choosing $\sigma_{q}^{2}$ is equivalent to choosing the scaling factor $\alpha$ in Figure 1(a). Since $\Gamma$ is assumed finite (which is consistent with the assumptions $\left.V, \sigma_{\bar{q}}^{2} \in \mathbb{R}^{+}\right), \sigma_{q}^{2}=0$ (equivalently, $\alpha=0$ ) is not an admissible choice in SNR constrained AWN channels. We will refer to $\gamma$ in (3) as the (stationary) channel SNR.

Remark 4 SNR constrained $A W N$ channels also arise in situations different from those described above. Indeed, [29] shows that certain erasure channels can be exactly modelled (as far as second order properties are concerned) as AWN channels with an SNR constraint that depends on the data-loss probability. On the other hand, [28] shows that for a certain class of source coding schemes (i.e., quantization schemes), average data rate constraints can be posed as SNR constraints in an AWN channel. We thus conclude that the study of SNR constrained AWN channels will yield insights into different NCS problems.

\section{Problem Definition}

In this paper we focus on the NCS depicted in Figure 2(a). In that figure, $\bar{P}$ is a generalized plant (see, e.g., [36]), $d$ is a disturbance signal, $e$ is a signal related to closed loop performance, $\bar{y}$ is a measurement that the controller $K$ uses to generate the plant input $\bar{u}$, and $q$ corresponds to noise in a scalar SNR constrained AWN channel.

The scheme of Figure 2(a) can be re-written as in Figure $2(\mathrm{~b})$, where

$$
\left[\begin{array}{c}
e \\
v \\
\hline \bar{y}
\end{array}\right]=P\left[\begin{array}{c}
d \\
q \\
\hline \bar{u}
\end{array}\right], \quad P \triangleq\left[\begin{array}{c|c}
P_{e 11} & P_{e 12} \\
P_{v 11} & P_{v 12} \\
\hline P_{21} & P_{22}
\end{array}\right] .
$$

Also define $P_{11} \triangleq\left[\begin{array}{ll}P_{e 11}^{T} & P_{v 11}^{T}\end{array}\right]^{T}$ and $P_{12} \triangleq\left[\begin{array}{ll}P_{e 12}^{T} & P_{v 12}^{T}\end{array}\right]^{T}$.

Assumption 5 The feedback system in Figure 2(b) is such that:

(a) The generalized plant $P$ belongs to $\mathcal{R}_{p}$, is stabilizable and is such that $P_{22} \in \mathcal{R}_{s p}$ (see (4)). 


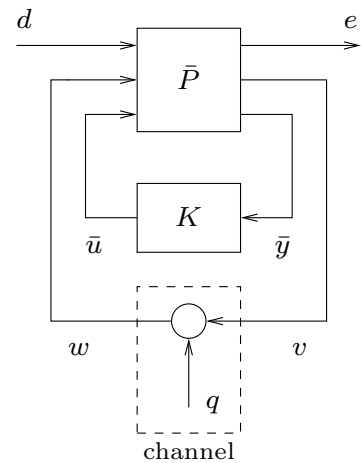

(a)

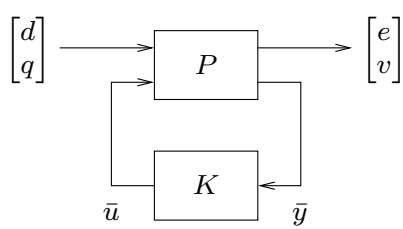

(b)
Fig. 2. (a) NCS closed over additive noise channel and (b) equivalent re-writting.

(b) $d$ is a second order wss process that admits a spectral factor $\Omega_{d} \in \mathcal{U}_{\infty}$, and is uncorrelated with $q$.

(c) The initial states of both $P$ and $K$ are jointly second order random variables, uncorrelated with $(d, q)$.

Our assumptions are mostly standard, except for the third part of $(a)$ which is non-essential, but simplifies matters.

In the sequel, we adopt the notion of mean square stability, as defined in, e.g., [7]:

Definition 6 Consider the linear system $x(k+1)=$ $A x(k)+B w(k)$, where $k \in \mathbb{N}_{0}, A, B$ are constant matrices of appropriate dimensions, $x(k) \in \mathbb{R}^{n}$ is the system state at time instant $k, x(0)=x_{o}, x_{o}$ is a second order random variable, and the input $w$ is a second order wss process uncorrelated with $x_{o}$. The system is said to be mean square stable (MSS) if and only if there exist finite $\mu \in \mathbb{R}^{n}$ and finite $M \in \mathbb{R}^{n \times n}, M \geq 0$, such that ${ }^{1}$

$$
\lim _{k \rightarrow \infty} \mathcal{E}\{x(k)\}=\mu, \quad \lim _{k \rightarrow \infty} \mathcal{E}\left\{x(k) x(k)^{T}\right\}=M,
$$

regardless of the initial state $x_{0}$.

It is well-known [2] that, for linear systems, MSS is equivalent to internal stability (in the standard sense [36]).

This paper studies the interplay between constraints on the stationary channel SNR, and the MSS and performance of the NCS depicted in Figure 2(a). To that end, we adopt the stationary variance $\sigma_{e}^{2}$ of $e$ as performance measure $^{2}$ and recall that, in our setup, the designer has the freedom to choose both $K \in \mathcal{R}_{p}$ and $\sigma_{q}^{2} \in \mathbb{R}^{+}$(equivalently, $\alpha \in \mathbb{R}^{+}$). We also denote by $T_{x y}$ the transfer function from any signal $x$ to another signal $y$ in Figure $2(\mathrm{~b})$.

$\overline{{ }^{1} \mathcal{E}\{\cdot\}}$ denotes the expectation operator.

2 Our results can be readily extended to other (weighted) quadratic performance measures.

\section{Mean square stability}

Subject to Assumption 5, and provided $\sigma_{q}^{2} \in \mathbb{R}^{+}$, the NCS of Figure 2(a) is MSS if and only if $K \in \mathcal{S}$, where

$$
\begin{array}{r}
\mathcal{S} \triangleq\left\{K \in \mathcal{R}_{p}\right. \text { : the feedback loop of Figure 2(b) } \\
\text { is internally stable and well-posed }\} .
\end{array}
$$

We use the well-known Youla parametrization to characterize $\mathcal{S}$ :

Theorem 7 (See, e.g., $[\mathbf{9}, \mathbf{3 5}, \mathbf{3 6}])$ Consider the feedback system of Figure 2(b) and assume that $P$ satisfies Assumption 5(a). Consider a doubly coprime factorization of $P_{22}$ over $\mathcal{R} \mathcal{H}_{\infty}$, i.e., consider $X_{i}, Y_{i}, X_{d}, Y_{d}, N_{i}$, $D_{i}, N_{d}, D_{d} \in \mathcal{R} \mathcal{H}_{\infty}$, with $X_{i}, X_{d}, D_{i}, D_{d}$ biproper, such that $P_{22}=N_{d} D_{d}^{-1}=D_{i}^{-1} N_{i}$ and

$$
\left[\begin{array}{cc}
X_{i} & -Y_{i} \\
-N_{i} & D_{i}
\end{array}\right]\left[\begin{array}{cc}
D_{d} & Y_{d} \\
N_{d} & X_{d}
\end{array}\right]=\left[\begin{array}{ll}
I & 0 \\
0 & I
\end{array}\right]
$$

Then, $K \in \mathcal{S}$ if and only if

$$
K=\left(X_{i}-Q N_{i}\right)^{-1}\left(Y_{i}-Q D_{i}\right),
$$

where $Q \in \mathcal{R H}_{\infty} \cdot{ }^{3}$ All transfer functions $T_{\bar{d} \bar{e}}$ from $\bar{d} \triangleq$ $\left[\begin{array}{ll}d^{T} & q^{T}\end{array}\right]^{T}$ to $\bar{e} \triangleq\left[\begin{array}{ll}e^{T} & v^{T}\end{array}\right]^{T}$ that are achievable with $K \in \mathcal{S}$ can be $K \in \mathcal{S}$ can be written as

$$
T_{\bar{d} \bar{e}}=T_{\bar{d} \bar{e}}^{o}-P_{12} D_{d} Q D_{i} P_{21},
$$

where $Q$ is as above, and $T_{\bar{d} \bar{e}}^{o} \triangleq P_{11}+P_{12} D_{d} Y_{i} P_{21}$. Moreover, $T_{\bar{d} \bar{e}}^{o}, P_{12} D_{d}$ and $D_{i} P_{21}$ belong to $\mathcal{R} \mathcal{H}_{\infty}$.

Define $J(Q) \triangleq\left\|M_{1}+M_{2} Q M_{3}\right\|_{2}^{2}$, where $Q \in \mathcal{R} \mathcal{H}_{\infty}$ is the decision variable and $M_{i} \in \mathcal{R} \mathcal{H}_{\infty}$ are given transfer functions. It is always possible to write $J(Q)=\mu+$ $\left\|A-B_{o} \operatorname{vec}\{Q\}\right\|_{2}^{2}$, where $\mu \in \mathbb{R}_{0}^{+}$is independent of $Q$, $A \in \mathcal{R H}_{\infty}$ and $B_{o}$ is outer (see [18] and $[9,34,35]$ ). $J_{\text {opt }} \triangleq \inf _{Q \in \mathcal{R H}_{\infty}} J(Q)$ always exists and is finite, but may not be achievable with $Q \in \mathcal{R H}_{\infty}[35$, Section 6.3]. In particular, $J_{o p t}$ is achievable if $B_{o}$ has full row rank on the unit circle. However, there always exists $Q_{\text {opt }} \in \overline{\mathcal{R H}}_{\infty}$ such that $J_{\text {opt }}=J\left(Q_{\text {opt }}\right)$. If $Q_{\text {opt }} \notin \mathcal{R} \mathcal{H}_{\infty}$, then the definition of inf guarantees the existence of a family of functions in $\mathcal{R} \mathcal{H}_{\infty}$, parameterized by $\varepsilon \in$ $(0,1]$ and with elements denoted by $\mathcal{K}_{\varepsilon}\left\{Q_{o p t}\right\}$, such that $J_{\text {opt }}=\lim _{\epsilon \rightarrow 0^{+}} J\left(\mathcal{K}_{\varepsilon}\left\{Q_{\text {opt }}\right\}\right.$ ) (see the proof of Lemma 10 in p. 171 of [35]). For convenience, we use the notation $\arg \inf _{Q \in \mathcal{R} \mathcal{H}_{\infty}} J(Q)$ to refer to any $Q_{\text {opt }}$ with the above properties. We also make $\mathcal{K}_{\varepsilon}\left\{Q_{o p t}\right\}=Q_{o p t}$ whenever $Q_{\text {opt }} \in \mathcal{R} \mathcal{H}_{\infty}$.

\footnotetext{
${ }^{3}$ Since $P_{22} \in \mathcal{R}_{s p}$, it suffices to pick $Q \in \mathcal{R} \mathcal{H}_{\infty}$ (see, e.g., $[35$, Section 5.2]).
} 
Using Theorem 7 we conclude that all transfer functions $T_{q v}$ from $q$ to $v$ in Figure 2(b) that are achievable with $K \in \mathcal{S}$ can be written as

$$
T_{q v}=T_{q v}^{o}-P_{v 12} D_{d} Q D_{i} P_{21} \eta
$$

where $T_{q v}^{o} \triangleq P_{v 11} \eta+P_{v 12} D_{d} Y_{i} P_{21} \eta$, and $\eta \triangleq\left[\begin{array}{llll}0 & \cdots & 0 & 1\end{array}\right]^{T}$.

Lemma 8 Consider the NCS of Figure 2(a), where $q$ is the noise in an SNR constrained AWN channel, and suppose that Assumption 5 holds. Define $E \triangleq\left(D_{i} P_{21} \eta\right)^{T} \otimes$ $\left(P_{v 12} D_{d}\right)$, where all transfer functions are as defined above. Factorize $E$ as $E=E_{i} E_{o}$, where $E_{i}$ is inner and $E_{o}$ is outer [9], and define $H \triangleq E_{i}^{\sim} T_{q v}^{o}$. Then:

(1) The minimal SNR compatible with MSS, say $\gamma_{\mathrm{inf}}$, is given by

$$
\gamma_{\text {inf }} \triangleq \inf _{\substack{Q \in \mathcal{R} \mathcal{H}_{\infty} \\ \sigma_{q}^{2} \in \mathbb{R}^{+}}} \gamma=\inf _{Q \in \mathcal{R} \mathcal{H}_{\infty}}\left\|T_{q v}\right\|_{2}^{2},
$$

and

$$
\begin{aligned}
Q_{\text {inf }} & \triangleq \underset{Q \in \mathcal{R} \mathcal{H}_{\infty}}{\arg \inf }\left\|T_{q v}\right\|_{2}^{2} \\
& =\operatorname{vec}^{-1}\left\{E_{o}^{\dagger}\left(\left.\left\{[H]_{\mathcal{H}_{2}^{\perp}}\right\}\right|_{z=0}+[H]_{\mathcal{H}_{2}}\right)\right\},
\end{aligned}
$$

where $E_{o}^{\dagger}$ is any right inverse of $E_{o}$ in $\overline{\mathcal{R H}}_{\infty}$.

(2) $\gamma_{\mathrm{inf}}$ is achievable if $P_{22}$ has no poles on the unit circle, both $P_{v 12}$ and $P_{21} \eta$ have no zeros on the unit circle, and the transfer function $T_{d v}$ between $d$ and $v$ is zero when $Q=Q_{\mathrm{inf}}$. If that is the case, then it suffices to choose $Q=Q_{\mathrm{inf}}$ and any $\sigma_{q}^{2} \in \mathbb{R}^{+}$.

(3) If $\gamma_{\mathrm{inf}}$ is not achievable, then one can cause $\gamma \rightarrow \gamma_{\mathrm{inf}}$ by choosing $Q=\mathcal{K}_{\varepsilon}\left\{Q_{\text {inf }}\right\}$ with $\varepsilon \rightarrow 0^{+}$, and $\sigma_{q}^{2}$ as follows: if $\left.T_{d v}\right|_{Q=Q_{\mathrm{inf}}}=0$, then choose any $\sigma_{q}^{2} \in \mathbb{R}^{+}$; otherwise, let $\sigma_{q}^{2} \rightarrow \infty$.

PROOF. Figure 2(b) and the definition on the left hand side of (3) imply that, for $K \in \mathcal{S}$ and $\sigma_{q}^{2} \in \mathbb{R}^{+}$,

$$
\gamma=\frac{\left\|T_{d v} \Omega_{d}\right\|_{2}^{2}}{\sigma_{q}^{2}}+\left\|T_{q v}\right\|_{2}^{2} \geq\left\|T_{q v}\right\|_{2}^{2}
$$

from where (8) follows. Define the unitary matrix $\phi \triangleq$ $\left[\left(I-E_{i} E_{i}^{\sim}\right)^{T}\left(E_{i}^{\sim}\right)^{T}\right]^{T}$. Use of (7), properties of the 2-norm (see, e.g., [18]), and standard analytic $\mathcal{H}_{2}$ optimization techniques (see, e.g., [34]) yield

$$
\begin{aligned}
\left\|T_{q v}\right\|_{2}^{2} & =\left\|\phi\left(T_{q v}^{o}-E_{i} E_{o} \operatorname{vec}\{Q\}\right)\right\|_{2}^{2} \\
& =\mu_{1}+\left\|\left.\left\{[H]_{\mathcal{H}_{2}^{\perp}}\right\}\right|_{z=0}+[H]_{\mathcal{H}_{2}}-E_{o} \operatorname{vec}\{Q\}\right\|_{2}^{2},
\end{aligned}
$$

where $\mu_{1} \in \mathbb{R}_{0}^{+}$is independent of $Q$. The remaining claims follow immediately from (9), (10), the definition of $E$, and properties of outer functions (see [9]).

Lemma 8 states that finding the minimal channel SNR that is compatible with MSS, namely $\gamma_{\mathrm{inf}}$, can be recast as the problem of minimizing the 2-norm of the transfer function $T_{q v}$. It is possible to use the techniques in, e.g., $[5,34]$, to provide an explicit analytic characterization of $\gamma_{\text {inf }}$. However, unless some structure is imposed on $P$, these characterizations are not easy to interpret. An explicit characterizations of $\gamma_{\text {inf }}$ will be given in Section 7 for specific NCSs.

Unless special conditions are satisfied, approaching $\gamma_{\text {inf }}$ requires a noise $q$ with arbitrarily large variance. (In the context of Section 3, this is tantamount to requiring an arbitrarily large scaling factor $\alpha$.) As a consequence, achieving MSS at SNRs close to $\gamma_{\text {inf }}$ compromises performance. This fact, which is consistent with results in [14] (see also [20]), motivates the study of the best performance that is achievable with a given SNR constraint $\Gamma$. It is also worth noting that having the freedom to choose $\sigma_{q}^{2}$ (equivalently, $\alpha$ ) is key to being able to achieve SNRs arbitrarily close to $\gamma_{\text {inf }}$ for all disturbances $d$. If $\sigma_{q}^{2}$ were fixed (e.g., by choosing $\alpha=1$ ), then the minimal SNR compatible with MSS would be, in general, strictly greater than $\gamma_{\text {inf }}$ unless $d=0$. This fact is also implicitly stated in [12, Section II.B].

\section{Performance}

We next study the best achievable performance in the scheme of Figure 2(a), when the channel is subject to an SNR constraint. We are interested in finding, for a given $\Gamma \in \mathbb{R}^{+}$,

$$
\left[\sigma_{e}^{2}\right]_{\Gamma} \triangleq \inf _{\substack{K \in \mathcal{S} \\ \sigma_{q}^{2} \in \mathbb{R}^{+} \\ \gamma \leq \Gamma}} \sigma_{e}^{2}
$$

It follows from Figure 2(b) that, if $K \in \mathcal{S}, \sigma_{q}^{2} \in \mathbb{R}^{+}$and Assumption 5 holds, then both $\sigma_{e}^{2}$ and $\sigma_{v}^{2}$ exist, are finite and satisfy

$$
\sigma_{e}^{2}=\left\|T_{\bar{d} e} \Omega_{\bar{d}}\right\|_{2}^{2}, \quad \sigma_{v}^{2}=\left\|T_{\bar{d} v} \Omega_{\bar{d}}\right\|_{2}^{2},
$$

where $\bar{d}$ is as in Theorem 7 and $\Omega_{\bar{d}} \triangleq \operatorname{diag}\left\{\Omega_{d}, \sigma_{q}\right\}$. All transfer functions $T_{\bar{d} e}$ and $T_{\bar{d} v}$ that are achievable with $K \in \mathcal{S}$ can be written as (see Theorem 7 )

$$
\begin{aligned}
& T_{\bar{d} e}=T_{\overline{d e}}^{o}-P_{e 12} D_{d} Q D_{i} P_{21}, \\
& T_{\bar{d} v}=T_{\bar{d} v}^{o}-P_{v 12} D_{d} Q D_{i} P_{21},
\end{aligned}
$$


where $T_{\bar{d} e}^{o} \triangleq P_{e 11}+P_{e 12} D_{d} Y_{i} P_{21}, T_{\bar{d} v}^{o} \triangleq P_{v 11}+$ $P_{v 12} D_{d} Y_{i} P_{21}$, and $Q$ is a free parameter in $\mathcal{R} \mathcal{H}_{\infty}$. Thus, (11) becomes equivalent to

$$
\left[\sigma_{e}^{2}\right]_{\Gamma}=\inf _{\sigma_{q}^{2} \in \mathbb{R}^{+}} \inf _{\substack{Q \in \mathcal{R} \mathcal{H} \\ R_{\sigma_{q}^{2}}(Q) \leq \Gamma \sigma_{q}^{2}}} J_{\sigma_{q}^{2}}(Q),
$$

where

$$
J_{\sigma_{q}^{2}}(Q) \triangleq\left\|T_{\bar{d} e} \Omega_{\bar{d}}\right\|_{2}^{2}, \quad R_{\sigma_{q}^{2}}(Q) \triangleq\left\|T_{\bar{d} v} \Omega_{\bar{d}}\right\|_{2}^{2} .
$$

Here, $J_{\sigma_{q}^{2}}(Q)$ is the stationary variance of $e$ expressed as a function of $Q$ with $\sigma_{q}^{2}$ as a parameter. Analogously, $R_{\sigma_{q}^{2}}(Q)$ denotes the corresponding stationary variance of $v$. We also define ${ }^{4}$

$$
\left(\sigma_{\Gamma}^{2}, Q_{\Gamma}\right) \triangleq \underset{\substack{Q \in \mathcal{R} \mathcal{H}_{\infty} \\ \sigma_{q}^{2} \in \mathbb{R}^{+} \\ R_{\sigma_{q}^{2}}(Q) \leq \Gamma \sigma_{q}^{2}}}{\operatorname{arginf}} J_{\sigma_{q}^{2}}(Q) .
$$

We will assume that the following holds (see also [18, p.17])

Assumption 9 The plant transfer functions $P_{21}^{T}, P_{v 12}$ and $P_{e 12}$ in (4) have full column normal rank. ${ }^{5}$

Assumption 9 and Assumption 5(b) ensure that both $J_{\sigma_{q}^{2}}$ and $R_{\sigma_{q}^{2}}$ are strictly convex functions of $Q$ for every fixed $\sigma_{q}^{2} \in \mathbb{R}^{+}$. These assumptions can be removed at the expense of more involved notation.

We are now ready to solve the problems in (13) and (15). Define, for every fixed $\sigma_{q}^{2} \in \mathbb{R}^{+}$, the set $\mathcal{P}_{\sigma_{q}^{2}}$ containing all Pareto-optimal points of the vector valued criterion $\left[\begin{array}{ll}J_{\sigma_{q}^{2}} & R_{\sigma_{q}^{2}}\end{array}\right]$ (see, e.g., [4, Section 4.7.3], [18]). Values of $\left(J_{\sigma_{q}^{2}}, R_{\sigma_{q}^{2}}\right) \in \mathcal{P}_{\sigma_{q}^{2}}$ provide the best trade-offs in the sense that there exist Youla parameters that achieve a smaller value for $J_{\sigma_{q}^{2}}$ at the expense of a smaller value for $R_{\sigma_{q}^{2}}$ for every feasible pair $\left(J_{\sigma_{q}^{2}}, R_{\sigma_{q}^{2}}\right)$ that does not belong to $\mathcal{P}_{\sigma_{q}^{2}}$. In our case, $\mathcal{P}_{\sigma_{q}^{2}}$ corresponds to a curve in $\mathbb{R}^{2}$, the optimal trade-off curve. The next lemma characterizes $\mathcal{P}_{\sigma_{q}^{2}}$ :

Lemma 10 Consider the feedback system of Figure 2(a) and suppose that Assumptions 5 and 9 hold.

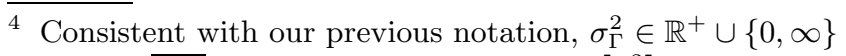
and $Q_{\Gamma} \in \overline{\mathcal{R H}}_{\infty}$ are such that $J_{\sigma_{\Gamma}^{2}}\left(Q_{\Gamma}\right)=\left[\sigma_{e}^{2}\right]_{\Gamma}$.

${ }^{5}$ i.e., full column rank for almost every $z \in \mathbb{C}$; see, e.g., [36].
(1) Define, for every $\sigma_{q}^{2} \in \mathbb{R}^{+}$and every $\epsilon \in[0,1]$,

$$
\begin{aligned}
L_{\sigma_{q}^{2}, \epsilon}(Q) & \triangleq \epsilon J_{\sigma_{q}^{2}}(Q)+(1-\epsilon) R_{\sigma_{q}^{2}}(Q), \\
Q_{\sigma_{q}^{2}, \epsilon} & \triangleq \arg \inf _{Q \in \mathcal{R} \mathcal{H}_{\infty}} L_{\sigma_{q}^{2}, \epsilon}(Q),
\end{aligned}
$$

where $J_{\sigma_{q}^{2}}$ and $R_{\sigma_{q}^{2}}$ are as in (14). Then, $\left(\alpha_{e}, \alpha_{v}\right) \in$ $\mathcal{P}_{\sigma_{q}^{2}}$ if and only if

$$
\left(\alpha_{e}, \alpha_{v}\right)=\left(J_{\sigma_{q}^{2}}\left(Q_{\sigma_{q}^{2}, \epsilon}\right), R_{\sigma_{q}^{2}}\left(Q_{\sigma_{q}^{2}, \epsilon}\right)\right)
$$

for some $\epsilon \in[0,1]$.

(2) Define

$$
A \triangleq\left[\begin{array}{c}
\sqrt{\epsilon} T_{\bar{d} e}^{o} \\
\sqrt{1-\epsilon} T_{\bar{d} v}^{o}
\end{array}\right] \Omega_{\bar{d}}, \quad E_{\epsilon} \triangleq\left[\begin{array}{c}
\sqrt{\epsilon} P_{e 12} \\
\sqrt{1-\epsilon} P_{v 12}
\end{array}\right],
$$

$A_{v} \triangleq \operatorname{vec}\{A\}, B_{v} \triangleq\left(D_{i} P_{21} \Omega_{\bar{d}}\right)^{T} \otimes\left(E_{\epsilon} D_{d}\right)$, where all symbols are as before, and factorize $B_{v}$ as $B_{v}=$ $B_{i} B_{o}$, where $B_{i}$ is inner and $B_{o}$ is outer [9]. Then, for any $\sigma_{q}^{2} \in \mathbb{R}^{+}$and $\epsilon \in[0,1]$, we have

$$
Q_{\sigma_{q}^{2}, \epsilon}=\operatorname{vec}^{-1}\left\{B_{o}^{-1}\left(\left.\left\{[H]_{\mathcal{H}_{2}^{\perp}}\right\}\right|_{z=0}+[H]_{\mathcal{H}_{2}}\right)\right\},
$$

where $H \triangleq B_{i}^{\sim} A_{v}$. Moreover, if $P_{22}$ has no poles on the unit circle and both $P_{21}$ and $E_{\epsilon}$ have no zeros on the unit circle, then $Q_{\sigma_{q}^{2}, \epsilon} \in \mathcal{R} \mathcal{H}_{\infty}$.

\section{PROOF.}

(1) Given our assumptions, $J_{\sigma_{q}^{2}}$ and $R_{\sigma_{q}^{2}}$ are strictly convex and, thus, so is $L_{\sigma_{q}^{2}, \epsilon}, \forall \epsilon \in[0,1]$. Accordingly, the optimization problem in (17) admits, at most, one solution (see $[4,19]$ ) and Theorem 2.1 in [18] yields the result.

(2) Using (16), (14), (12) and proceeding as in the proof of Lemma 8, it is possible to write

$$
\begin{aligned}
L_{\sigma_{q}^{2}, \epsilon}(Q)= & \left\|A_{v}-B_{v} \operatorname{vec}\{Q\}\right\|_{2}^{2} \\
=\mu_{2}+\|\left.\left\{[H]_{\mathcal{H}_{2}^{\perp}}\right\}\right|_{z=0} & \\
& \quad+[H]_{\mathcal{H}_{2}}-B_{o} \operatorname{vec}\{Q\} \|_{2}^{2},
\end{aligned}
$$

where $\mu_{2} \in \mathbb{R}_{0}^{+}$is independent of $Q$. Since $P_{21}^{T}, P_{e 12}$ and $P_{v 12}$ have full column normal rank, and $\Omega_{\bar{d}} \in$ $\mathcal{U}_{\infty}$, it is immediate to see that $B_{v}$ has full column normal rank [3, Fact 7.4.20]. Hence, the definition of outer function [9] implies that $B_{o} \in \overline{\mathcal{U}}_{\infty}$ and that all its zeros on the unit circle correspond to the zeros on the unit circle of $E_{\epsilon} D_{d}$ and $D_{i} P_{21} \Omega_{\bar{d}}$. Our claims are now immediate. $\left(B_{o} \in \mathcal{U}_{\infty}\right.$ is only sufficient for $Q_{\sigma_{q}^{2}, \epsilon} \in \mathcal{R} \mathcal{H}_{\infty}$.) 
Lemma 10 allows one to find, for every $\sigma_{q}^{2} \in \mathbb{R}^{+}$, an optimal trade-off curve in the $J_{\sigma_{q}^{2}}$ versus $R_{\sigma_{q}^{2}}$ plane or, equivalently, in the $\sigma_{e}^{2}$ versus $\gamma$ plane.

We now present an exact characterization of the best achievable performance $\left[\sigma_{e}^{2}\right]_{\Gamma}$ :

Theorem 11 Consider the setup of Figure 2(a), where $q$ is the noise in an AWN channel with SNR constraint $\Gamma$. Suppose that Assumptions 5 and 9 hold and that $\gamma_{\mathrm{inf}}<$ $\Gamma<\infty$. Define the set

$$
\Sigma_{\Gamma} \triangleq\left\{\sigma_{q}^{2} \in \mathbb{R}^{+}: \inf _{Q \in \mathcal{R} \mathcal{H}_{\infty}} R_{\sigma_{q}^{2}}(Q) \leq \Gamma \sigma_{q}^{2}\right\}
$$

and the function $f_{\Gamma}: \Sigma_{\Gamma} \rightarrow[0,1]$, where

$f_{\Gamma}\left(\sigma_{q}^{2}\right) \triangleq \begin{cases}1 & \text { if } \Gamma \sigma_{q}^{2}>R_{\sigma_{q}^{2}}\left(Q_{\sigma_{q}^{2}, 1}\right), \\ \epsilon & \text { if } \epsilon \in[0,1] \text { is such that } \Gamma \sigma_{q}^{2}=R_{\sigma_{q}^{2}}\left(Q_{\sigma_{q}^{2}, \epsilon}\right) .\end{cases}$

Then:

(1) The optimal noise variance $\sigma_{\Gamma}^{2}$ is given by

$$
\sigma_{\Gamma}^{2}=\underset{\sigma_{q}^{2} \in \Sigma_{\Gamma}}{\arg \inf } J_{\sigma_{q}^{2}}\left(Q_{\sigma_{q}^{2}, f_{\Gamma}\left(\sigma_{q}^{2}\right)}\right),
$$

and the optimal Youla parameter $Q_{\Gamma}$ and the minimal stationary variance $\left[\sigma_{e}^{2}\right]_{\Gamma}$ satisfy

$$
Q_{\Gamma}=Q_{\sigma_{\Gamma}^{2}, f\left(\sigma_{\Gamma}^{2}\right)}, \quad\left[\sigma_{e}^{2}\right]_{\Gamma}=J_{\sigma_{\Gamma}^{2}}\left(Q_{\Gamma}\right) .
$$

(2) If $P_{22}$ has no poles on the unit circle and both $P_{21}$ and $E_{f_{\Gamma}\left(\sigma_{\Gamma}^{2}\right)}$ (see (18)) have no zeros on the unit circle, then $Q_{\Gamma} \in \mathcal{R} \mathcal{H}_{\infty}$ and $\left[\sigma_{e}^{2}\right]_{\Gamma}$ can be achieved with an SNR no greater than $\Gamma$ upon choosing $\sigma_{q}^{2}=\sigma_{\Gamma}^{2}$ and $Q=Q_{\Gamma}$. In all other cases, it is possible to get arbitrarily close to $\left[\sigma_{e}^{2}\right]_{\Gamma}$, while violating the SNR constraint as little as desired, by choosing $\sigma_{q}^{2}=\sigma_{\Gamma}^{2}$ and $Q=\mathcal{K}_{\varepsilon}\left\{Q_{\Gamma}\right\}$ with $\varepsilon \rightarrow 0^{+}$.

PROOF. Since $\Gamma>\gamma_{\mathrm{inf}}$, the problem of finding $\left[\sigma_{e}^{2}\right]_{\Gamma}$ is feasible. It is also clear that the inner optimization problem in (13) is feasible if and only if $\inf _{Q \in \mathcal{R H}}$ $R_{\sigma_{q}^{2}}(Q) \leq \Gamma \sigma_{q}^{2}$. Thus, all $\sigma_{q}^{2}$ of interest lie in $\Sigma_{\Gamma}$. For a fixed $\sigma_{q}^{2} \in \Sigma_{\Gamma}$, define

$$
Q_{\sigma_{q}^{2}} \triangleq \underset{\substack{Q \in \mathcal{R} \mathcal{H}_{\infty} \\ R_{\sigma_{q}^{2}}(Q) \leq \Gamma \sigma_{q}^{2}}}{\arg \inf } J_{\sigma_{q}^{2}}(Q)
$$

Lemma 10, the strict convexity of both $J_{\sigma_{q}^{2}}$ and $R_{\sigma_{q}^{2}}$, and the definition of Pareto optimality imply that: (i) if $\Gamma \sigma_{q}^{2} \leq R_{\sigma_{q}^{2}}\left(Q_{\sigma_{q}^{2}, 1}\right)$, then $Q_{\sigma_{q}^{2}}$ is unique and $Q_{\sigma_{q}^{2}}=$ $Q_{\sigma_{q}^{2}, f_{\Gamma}\left(\sigma_{q}^{2}\right)}$; (ii) if $R_{\sigma_{q}^{2}}\left(Q_{\sigma_{q}^{2}, 1}\right)<\Gamma \sigma_{q}^{2}$, then, by convexity, $R_{\sigma_{q}^{2}}\left(Q_{\sigma_{q}^{2}, \epsilon}\right)<\Gamma \sigma_{q}^{2}$ for every $\epsilon \in[0,1]$ and the constraint on $R_{\sigma_{q}^{2}}$ becomes redundant. In those cases, one can choose $Q_{\sigma_{q}^{2}}=Q_{\sigma_{q}^{2}, 1}=Q_{\sigma_{q}^{2}, f_{\Gamma}\left(\sigma_{q}^{2}\right)}$. (Here, $Q_{\sigma_{q}^{2}}$ is not unique.)

We now return to (13). To solve (13), it suffices to pick the value of $\sigma_{q}^{2}$ that minimizes $J_{\sigma_{q}^{2}}\left(Q_{\sigma_{q}^{2}}\right)=$ $J_{\sigma_{q}^{2}}\left(Q_{\sigma_{q}^{2}, f_{\Gamma}\left(\sigma_{q}^{2}\right)}\right)$. This is achieved by solving the optimization problem in (19). Our remaining claims follow from Part 2 of Lemma 10.

Remark 12 From Lemma 8 we know that the optimization problem in (13) is infeasible if $\Gamma<\gamma_{\mathrm{inf}}$. If $T_{d v}$ or $T_{q e}$ are zero for $Q=Q_{\text {inf }}$ (see Lemma 8), or $\gamma_{\text {inf }}$ is achievable, then $\left[\sigma_{e}^{2}\right]_{\Gamma} \rightarrow J_{0}\left(Q_{\mathrm{inf}}\right)$ as $\gamma \rightarrow \gamma_{\mathrm{inf}}$. If, on the other hand, $\gamma_{\mathrm{inf}}$ is not achievable and both $T_{q e} \neq 0$ and $T_{d v} \neq 0$ for $Q=Q_{\mathrm{inf}}$, then $\left[\sigma_{e}^{2}\right]_{\Gamma} \rightarrow \infty$ when $\gamma \rightarrow \gamma_{\mathrm{inf}}$.

Remark 13 Using Lemma 10 and the definition of Pareto optimality, Theorem 11 can be modified so as to provide a characterization of the minimal channel SNR that allows one to achieve a given performance level.

Theorem 11 provides an analytic characterization of the best performance achievable with an SNR constraint $\Gamma$ in the NCS of Figure 2. Although analytical, our results are not explicit, but can be used as the basis of simple numerical algorithms to approximate $\left[\sigma_{e}^{2}\right]_{\Gamma}$, and to find the associated optimal noise variance and Youla parameter (see, e.g., Algorithm 1 in [29]).

We next present two properties of the solution to the problems in (13) and (15):

Lemma 14 Consider the setup and assumptions of Theorem 11. Assume that either $T_{d v}=0$ is not achievable with $Q \in \overline{\mathcal{R H}}_{\infty}$, or that $\inf _{Q \in \mathcal{R H}} J_{0}(Q)<J_{0}\left(Q^{0}\right)$, where $Q^{0} \in \overline{\mathcal{R H}}_{\infty}$ is such that $\left.T_{d v}\right|_{Q=Q^{0}}=0$. Then:

(1) If $\Gamma \neq\left\|T_{q v}^{0}\right\|_{2}^{2}$, where $\left.T_{q v}^{0} \triangleq T_{q v}\right|_{Q=Q^{0}}$, then $\sigma_{\Gamma}^{2} \neq 0$ and $\left.T_{d v}\right|_{Q=Q_{\Gamma}} \neq 0$.

(2) If $Q_{\Gamma}$ is such that $T_{q e} \neq 0$, then the inequality constraint in (13) is active at the optimum.

PROOF. The proof is not difficult, but omitted due to space constraints.

If $\inf _{Q \in \mathcal{R} \mathcal{H}_{\infty}} J_{0}(Q) \geq J_{0}\left(Q^{0}\right)$, then the use of even a perfect channel between $v$ and $w$ in Figure 2(a) brings no benefits to closed loop performance. This situation is of no interest in an NCS setting. Thus, Part 1 of Lemma 14 
guarantees, for almost every choice of $\Gamma$, a non zero optimal channel noise variance $\sigma_{\Gamma}^{2}$. (This is natural since we consider finite upper bounds on the channel SNR.) Consider now Part 2. If $T_{q e}=0$ at the optimum, then one would have an NCS where the effects of communication constraints on closed loop performance can be made zero while respecting the SNR constraint. Again, this situation is of little interest in NCSs. Thus, for most cases of interest, the SNR constraint will be active at the optimum.

\section{$7 \quad$ Specific Architectures}

In this section we study specific SNR constrained NCSs built around discrete-time LTI SISO plant models. We assume that one-step-delayed channel feedback is available, and focus on the setup illustrated in Figure 3. In that figure, $G$ is the plant, $r$ is a reference signal, $d_{o}$ is a disturbance, ${ }^{6} q$ is the noise in an SNR constrained AWN channel, $K_{1}$ is the encoder, and $K_{2}$ is the controller and decoder. The role of the encoder is to use the plant output $y$ and the channel output $w$ to construct the signal that is sent over the channel. The controller and decoder uses the reference signal $r$ and channel output $w$ to generate the plant input $u$.

The architecture of Figure 3 is based on feedback coding ideas (see, e.g., [17]), and generalizes the architectures in $[5,12,16,30,32]$. As will become clear below, the use of channel feedback is a key feature of the proposed architecture.

Remark 15 Perfect (but delayed) channel feedback may not be available in some practical settings. There exist, however, situations where this is a natural assumption. Recall from Section 3 that AWN channels can be used to model situations that include noiseless digital channels or channels prone to data loss. In the former case, knowing the channel input amounts to knowing its output [20]. In channels prone to data loss (but otherwise transparent; see, e.g., [26]), the use of packet acknowledgements is also equivalent to having ideal feedback around the channel.

Throughout this section we assume the following:

Assumption 16 The feedback architecture in Figure 3 is such that:

(a) $G$ is SISO, non-zero, and has a stabilizable and detectable underlying realization.

(b) $r$ and $d_{o}$ are second order mutually uncorrelated wss scalar processes that, if not zero, admit spectral factors in $\mathcal{U}_{\infty}$. At least one signal, $r$ or $d_{o}$, is non-zero. The channel noise $q$ is uncorrelated with $\left(r, d_{o}\right)$.

\footnotetext{
6 The inclusion of additional disturbance signals and measurement noise does not pose any difficulty.
}

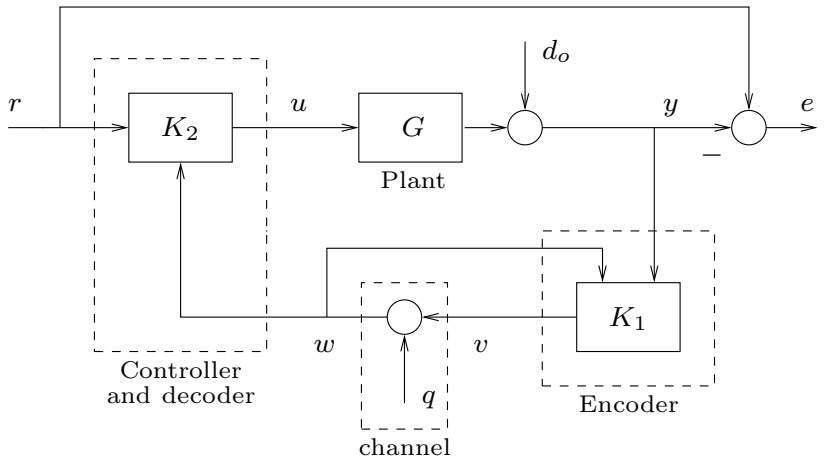

Fig. 3. A specific networked control architecture.

(c) The initial states of $K_{1}, K_{2}$ and $G$ are jointly second order random variables, and are uncorrelated with $\left(r, d_{o}, q\right)$.

Assumption 16 is standard, except for the fact that we require $r$ or $d_{o}$ to be non-zero for brevity.

Our interest lies in control architectures that use only LTI filters. This choice limits the performance of the resulting NCS, but opens the door to use linear control theoretic results, such as those presented in Sections 5 and 6 . Thus, we consider $K_{1} \triangleq\left[\begin{array}{ll}K_{11} & K_{12}\end{array}\right]$ and $K_{2} \triangleq\left[\begin{array}{ll}K_{21} & K_{22}\end{array}\right]$ to be design variables in $\mathcal{R}_{p}^{1 \times 2}$ that that satisfy ${ }^{7}$

$$
v=K_{11} w+K_{12} y, \quad u=K_{21} r+K_{22} w .
$$

We also define $\bar{K} \triangleq\left[\begin{array}{ll}K_{1} & K_{2}\end{array}\right]$, and recall that the signal $w$, if available at the encoder side, is available with one step delay. Accordingly, $K_{11} \in \mathcal{R}_{s p}$. This constraint will be enforced throughout the rest of the paper.

Re-define $\mathcal{S}$ as follows (cf. (5)):

$$
\mathcal{S} \triangleq\left\{\bar{K} \in \mathcal{R}_{p}^{1 \times 4}: K_{11} \in \mathcal{R}_{s p}\right. \text {, and the feedback loop }
$$
of Figure 3 is internally stable and well-posed $\}$.

Note that, when channel feedback is not available, then $K_{11}=0$ is a natural constraint on $\mathcal{S}$. Note also that the choice

$$
K_{11}=0, \quad K_{12}=1, \quad K_{21}=-K_{22}=C,
$$

with $C \in \mathcal{R}_{p}$, reduces the architecture of Figure 3 to a one degree-of-freedom (one-dof) NCS, as studied in $[5,12,32]$.

It follows from [5, Theorem III.2] and Lemma 8 that, provided Assumption 16 holds and the plant has only simple finite strictly-non-MP zeros, the minimal SNR

\footnotetext{
7 The $n \times m$ superscript emphasizes the dimension of $K_{1}$ and $K_{2}$.
} 
compatible with MSS in a one-dof architecture is given by

$$
\gamma_{\text {inf }}^{\text {one-dof }} \triangleq \inf _{\substack{\bar{K} \in \mathcal{S} \cap \mathcal{O} \\ \sigma_{q}^{2} \in \mathbb{R}^{+}}} \gamma=\left(\prod_{i=1}^{n_{p}}\left|p_{i}\right|^{2}\right)-1+\Delta_{G},
$$

where $\mathcal{O}$ is the set of all $\bar{K}$ satisfying $(21), p_{i}$ is the $i^{\text {th }}$ unstable plant pole, $\Delta_{G} \geq 0$ depends on the non-MP zeros and relative degree of $G$, and $\Delta_{G}=0$ if and only if the plant has no finite strictly-non-MP zeros and has relative degree one (see [5]).

As shown next, the architecture of Figure 3 allows one to reduce the minimal SNR compatible with MSS:

Theorem 17 Consider the feedback system of Figure 3, where $G$ is unstable, ${ }^{8} q$ is the noise in an SNR constrained AWN channel, and Assumption 16 holds. Then:

(1) If $\bar{K}$ can be chosen freely in $\mathcal{S}$, then

$$
\gamma_{\mathrm{inf}} \triangleq \inf _{\substack{\bar{K} \in \mathcal{S} \\ \sigma_{q}^{2} \in \mathbb{R}^{+}}} \gamma=\left(\prod_{i=1}^{n_{p}}\left|p_{i}\right|^{2}\right)-1 .
$$

(2) If no channel feedback is available, and $G$ has only simple finite strictly-non-MP zeros, ${ }^{9}$ then

$$
\gamma_{\text {inf }}^{\text {no-fb }} \triangleq \inf _{\substack{\bar{K} \in \mathcal{S} \cap \mathcal{W} \\ \sigma_{q}^{2} \in \mathbb{R}^{+}}} \gamma=\gamma_{\text {inf }}^{\text {one-dof }}
$$

where $\mathcal{W}$ is the set of all $\bar{K}$ with $K_{11}=0$, and $\gamma_{\text {inf }}^{\text {one-dof }}$ is as in $(22)$.

\section{PROOF.}

(1) Theorem 7 and other standard results (see, e.g., [36, Chapter 12]) imply that $\bar{K} \in \mathcal{S}$ if and only if: (i) $K_{2}$ has a detectable and stabilizable underlying realization such that the inherited realization of the transfer function between $v$ and $[w y]$ in Figure 3, when $K_{1}$ is removed, is detectable and stabilizable, and (ii) $K_{1}=\left(X_{i}-Q N_{i}\right)^{-1}\left(Y_{i}-Q D_{i}\right) \operatorname{diag}\left\{z^{-1}, 1\right\}$, where $Q, X_{i}, Y_{i}, N_{i}, D_{i}, N_{d}, D_{d}$ are as in Theorem 7 with $P_{22}=\left[\begin{array}{ll}z^{-1} & G K_{22}\end{array}\right]^{T}$.

Consider any $K_{2}$ as above, and $N, D \in \mathcal{R H}_{\infty}$, coprime in $\mathcal{R H}_{\infty}$ and with $D$ biproper, such that $G K_{22}=N D^{-1}$. Then, the following choices are possible $N_{d} \triangleq\left[\begin{array}{ll}z^{-1} D & N\end{array}\right]^{T}, D_{d} \triangleq D$,

\footnotetext{
8 If $G$ is stable then the minimal SNR compatible with MSS is zero for any architecture.

9 This part (and the results in [5]) can be extended to general unstable plants at the expense of more involved notation.
}

$N_{i} \triangleq\left[\begin{array}{ll}z^{-1} & N\end{array}\right]^{T}$ and $D_{i} \triangleq \operatorname{diag}\{1, D\}$. Thus, we can use use Theorem 7 to conclude that all transfer functions $T_{q v}$ from $q$ to $v$ in Figure 3 that are achievable with $\bar{K} \in \mathcal{S}$ are given by

$$
T_{q v}=Y_{i} N_{d}-D Q N_{i}=X_{i} D-1-D Q N_{i},
$$

where the second equality follows from (6). (We also note that (6) implies that, since $G \in \mathcal{R}_{s p}$ and $Y_{i} \in \mathcal{R H}_{\infty}, X_{i}(\infty) D(\infty)-1=Y_{i}(\infty) N_{d}(\infty)=0$.)

Since Assumption 16 holds, it suffices to find $\inf _{Q \in \mathcal{R} \mathcal{H}_{\infty}}\left\|T_{q v}\right\|_{2}^{2}$ (see Lemma 8). From (23),

$$
\begin{aligned}
& \left\|1-X_{i} D+D Q N_{i}\right\|_{2}^{2} \\
& \stackrel{(a)}{=}\left\|\xi_{p}-\xi_{p} X_{i} D+\xi_{p} D Q\left[\begin{array}{c}
z^{-1} \\
N
\end{array}\right]\right\|_{2}^{2} \\
& \stackrel{(b)}{=}\left\|\xi_{p}-\xi_{p}(0)\right\|_{2}^{2}+\left\|\xi_{p}(0)-\xi_{p}(\infty)\right\|_{2}^{2} \\
& \quad+\left\|\left(\xi_{p}(\infty)-X_{i} \xi_{p} D\right)+\xi_{p} D Q\left[\begin{array}{c}
z^{-1} \\
N
\end{array}\right]\right\|_{2}^{2} \\
& \stackrel{(c)}{=}\left(\prod_{i=1}^{n_{p}}\left|p_{i}\right|^{2}\right)-1 \\
& +\left\|z\left(\xi_{p}(\infty)-X_{i} \xi_{p} D\right)+\xi_{p} D Q\left[\begin{array}{c}
1 \\
z N
\end{array}\right]\right\|_{2}^{2},
\end{aligned}
$$

where $\xi_{p} \triangleq \prod_{i=1}^{n_{p}} \frac{1-z p_{i}}{z-p_{i}}$. In (24), (a) follows from the definition of $N_{i}$, the fact that $\xi_{p}$ is unitary and properties of the 2 -norm, (b) follows using standard analytic $\mathcal{H}_{2}$ optimization techniques based on orthogonal decompositions (see, e.g., $[5,34]$ ), and (c) follows from the fact that $z$ is unitary and from the residue theorem (see, e.g., Appendix $A$ in [27]). Thus,

$$
\gamma \stackrel{(a)}{\geq}\left\|T_{q v}\right\|_{2}^{2} \stackrel{(b)}{\geq}\left(\prod_{i=1}^{n_{p}}\left|p_{i}\right|^{2}\right)-1,
$$

for every $\bar{K} \in \mathcal{S}$ and every $\sigma_{q}^{2} \in \mathbb{R}^{+}$. The gap between the left and right hand sides of $(a)$ in $(25)$ can be made arbitrary small by choosing $\sigma_{q}^{2}$ sufficiently large (see proof of Lemma 8). On the other hand, choosing $Q=\mathcal{K}_{\varepsilon}\left\{-z\left(\xi_{p} D\right)^{-1}\left(\xi_{p}(\infty)-X_{i} \xi_{p} D\right) \times\right.$ $\left.\left[\begin{array}{ll}1 & 0\end{array}\right]\right\}$, with a sufficiently small $\varepsilon \in(0,1]$, guarantees that the gap between both sides of $(b)$ in $(25)$ is arbitrarily small. The result is now immediate.

(2) This part follows proceeding as above and using the proof of Theorem III.2 in [5].

Remark 18 An expression for the filter $\bar{K} \in \mathcal{S}$ (or $\bar{K} \in$ $\mathcal{S} \cap \mathcal{W}$ ) and the channel variance $\sigma_{q}^{2} \in \mathbb{R}^{+}$that allows one to achieve any SNR arbitrarily close to $\gamma_{\mathrm{inf}}$ (or $\gamma_{\mathrm{inf}}^{\mathrm{no}-\mathrm{fb}}$ ) 
follows immediately from the proof of Theorem 17 and Lemma 8.

Part 1 of Theorem 17 states that, when the proposed architecture is employed, the minimal SNR compatible with MSS becomes a function of the unstable plant poles only. This stands in contrast to the one-dof architecture studied in [5], where plant non-MP zeros and relative degree play a role. The control architecture proposed here thus has the ability to enlarge the class of plants that are stabilizable over a given SNR constrained AWN channel. The key feature of the proposed architecture is that it exploits channel feedback and, accordingly, uses additional degrees of freedom not available in a one-dof control architecture. (Our conclusions are thus akin to those in, e.g., [6, Section IV], where it is shown that additional degrees of freedom are fundamental to circumvent limitations imposed by plant non-MP zeros or unstable poles.) The latter is reinforced by the second part of our result: When no channel feedback is available (equivalently, $\left.K_{11}=0\right)$ then, no matter how many additional degrees of freedom are employed, the minimal SNR required for MSS is as in a one-dof architecture.

Remark 19 If $K_{2} \in \mathcal{R} \mathcal{H}_{\infty}$ is fixed and satisfies the conditions at the beginning of the proof of Theorem 17, then Part 1 of the result still applies. Also, if $K_{1}=\left[\begin{array}{ll}0 & K_{12}\end{array}\right]$ and $K_{12} \in \mathcal{U}_{\infty}$ is any fixed transfer function with stabilizable and detectable underlying realization, and such that the inherited realization of the transfer function between $u$ and $w$ in Figure 3, when $K_{2}$ is removed, is detectable and stabilizable, then Part 2 of Theorem 17 also applies.

Assume now that the performance of the architecture of Figure 3 is measured by means of the stationary variance $\sigma_{e}^{2}$ of the tracking error $e \triangleq r-y$. In principle, once Figure 3 is arranged as in Figure 2(b), and provided Assumption 16 holds, the results of Section 6 yield a characterization of the minimal stationary tracking error variance that is achievable with a given SNR constraint. This is true if $\bar{K}$ is constrained as in (21), or if either $K_{1}$ or $K_{2}$ are fixed and the remaining transfer function has to be designed.

Unfortunately, the situation is not that simple when no constraints are imposed on $\bar{K}$ or when only $K_{11}=0$ is enforced. In those cases, $u$ cannot depend on $y$ and $v$ cannot depend on $r$ (see Figure 3). Hence, the equivalent controller $K$ in Figure 2(b) has entries that are identically zero. Thus, the optimal design of $K$ becomes an optimal control problem with sparsity constraints (see, e.g., $[25,31])$. Such problems are, in general, non-convex and difficult to solve [33]. In these cases, we propose to use the results of Section 6 to design $K$ in an iterative fashion: Fix $K_{1}$ (or $K_{2}$ ) and choose $K_{2}$ (resp. $K_{1}$ ) so as to optimize performance subject to the SNR constraint $\Gamma$. For that choice of $K_{2}\left(\right.$ resp. $\left.K_{1}\right)$ choose $K_{1}\left(\right.$ resp. $\left.K_{2}\right)$ in a similar fashion, etc. The procedure outlined converges, at least, to a local minimum.

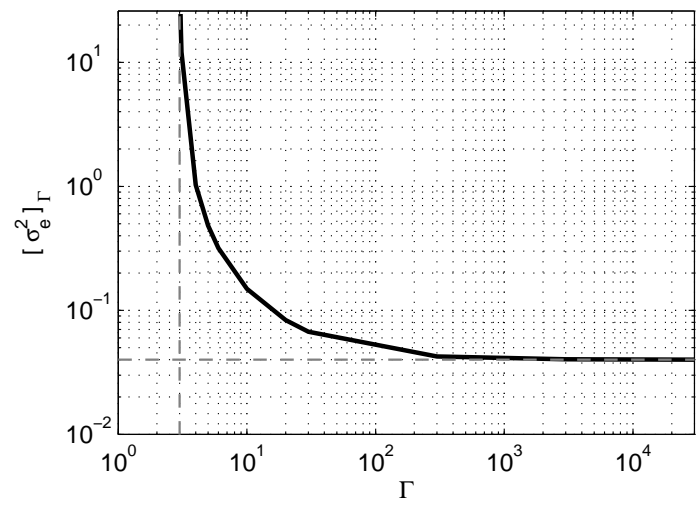

Fig. 4. Optimal performance $\left[\sigma_{e}^{2}\right]_{\Gamma}$ as a function of upper bound $\Gamma$ on the channel SNR.

\section{Example}

Consider the unstable plant model

$$
G=\frac{z-0.8}{z(z-2)}
$$

assume that $d_{o}=0$, and that the reference $r$ has a spectral factor $\Omega_{r}=0.1 z(z-0.9)^{-1}$. We will design a onedof NCS architecture (see (21)) so as to minimize the stationary tracking error variance in the face of an SNR constraint. For this plant and architecture, the minimal SNR compatible with MSS is given by $\gamma_{\text {inf }}^{\text {one-dof }}=3$.

We have computed $\left[\sigma_{e}^{2}\right]_{\Gamma}$ for several values of $\Gamma \in$ $\left(3,3 \cdot 10^{4}\right]$. The results are shown in Figure 4 . As expected, $\left[\sigma_{e}^{2}\right]_{\Gamma}$ is a decreasing function of $\Gamma$ that tends to the best achievable non-networked performance $D_{\text {inf }} \triangleq \inf _{Q \in \mathcal{R} \mathcal{H}_{\infty}} J_{0}(Q)=0.04$ as $\Gamma \rightarrow \infty$, and that grows unbounded when $\Gamma \rightarrow 3$ (see horizontal and vertical lines in Figure 4, respectively). For moderate values of $\Gamma$, the best achievable performance is almost identical to $D_{\text {inf }}$.

\section{Conclusions}

This paper has presented a framework within which one can study control problems with SNR constraints. We have focused on the problem of elucidating the interplay between closed loop performance and SNR constraints and, in particular, we have provided a characterization of the best achievable performance subject to a given SNR constraint. We have also presented a detailed study of the minimal SNR needed to achieve MSS in two architectures of interest. Our results show that the bound on the channel SNR required to achieve MSS presented in [5] for the noiseless static state feedback case, can be recovered with noisy output feedback, and for any plant model and any combination of external noise sources, provided channel feedback is available. 
Possible extensions of the results presented in this paper lie in the consideration of MIMO plant models, and of architectures with multiple channels (see preliminary results in [21]). The search for explicit analytical characterizations of the best performance achievable with a given SNR constraint are also of interest.

\section{Acknowledgements}

The first author acknowledges the support from CONICYT through grant FONDECYT 3100024.

\section{References}

[1] P. Antsaklis and J. Baillieul. Special issue on technology of networked control systems. Proceedings of the IEEE, 95(1):58, 2007.

[2] K.J. Åström. Introduction to Stochastic Control Theory. Academic Press, 1970.

[3] D.S. Bernstein. Matrix Mathematics. Princeton University Press, 2005.

[4] S. Boyd and L. Vandenberghe. Convex Optimization. Cambridge University Press, 2004.

[5] J.H. Braslavsky, R.H. Middleton, and J.S. Freudenberg. Feedback stabilization over signal-to-noise ratio constrained channels. IEEE Transactions on Automatic Control, 52(8):1391-1403, 2007.

[6] J. Chen, L. Qiu, and O. Toker. Limitations on maximal tracking accuracy. IEEE Transactions on Automatic Control, 45(2):326-331, 2000.

[7] O.L.V. Costa, M.D. Fragoso, and R.P. Marques. Discrete Time Markov Jump Linear Systems. Springer, 2005.

[8] T.M. Cover and J.A. Thomas. Elements of Information Theory. John Wiley and Sons, 2006.

[9] B. Francis. A Course on $H_{\infty}$ Control Theory. Springer, 1987.

[10] J.S. Freudenberg, J.H. Braslavsky, and R.H. Middleton. Control over signal-to-noise ratio constrained channels: Stabilization and performance. In Proceedings of the 44th IEEE Conference on Decission and Control and ECC, 2005.

[11] J.S. Freudenberg and R.H. Middleton. Feedback control performance over a noisy communication channel. In Proceedings of the Information Theory Workshop, 2008.

[12] J.S. Freudenberg, R.H. Middleton, and J.H. Braslavsky. Stabilization with disturbance attenuation over a Gaussian channel. In Proceedings of the 46th IEEE Conference on Decision and Control, 2007.

[13] J.S. Freudenberg, R.H. Middleton, and J.H. Braslavsky. Minimum variance control over a gaussian communication channel. In American Control Conference, 2008.

[14] J.S. Freudenberg, R.H. Middleton, and V. Solo. The minimal signal-to-noise ratio requirements to stabilize over a noisy channel. In Proceedings of the American Control Conference, 2006.

[15] A. Goldsmith. Wireless Communications. Cambridge University Press, 2005.

[16] G.C. Goodwin, D.E. Quevedo, and E.I. Silva. Architectures and coder design for networked control systems. Automatica, 44(1):248-257, 2008.
[17] N. Jayant and P. Noll. Digital Coding of Waveforms. Principles and Approaches to Speech and Video. Prentice Hall, 1984.

[18] P.P. Khargonekar and M.A. Rotea. Multiple objective optimal control of linear systems: the quadratic norm case. IEEE Transactions on Automatic Control, 36(1):14-24, 1991.

[19] D. Luenberger. Optimization by Vector Space Methods. John Wiley and Sons, 1969.

[20] G. Nair and R. Evans. Stabilizability of stochastic linear systems with finite feedback data rates. SIAM Journal on Control and Optimization, 43(2):413-436, 2004.

[21] D.E. Quevedo, E.I. Silva, and G.C. Goodwin. Subband coding for networked control systems. International Journal of Robust and Nonlinear Control, 19:1817-1836, 2009.

[22] A.J. Rojas, J.H. Braslavsky, and R.H. Middleton. Output feedback stabilisation over bandwidth limited, signal to noise ratio constrained communication channels. In Proceedings of the American Control Conference, 2006.

[23] A.J. Rojas, J.H. Braslavsky, and R.H. Middleton. Output feedback sensitivity functions under signal to noise ratio constraint. In Proceedings of the American Control Conference, 2007.

[24] A.J. Rojas, J.H. Braslavsky, and R.H. Middleton. Channel signal-to-noise ratio constrained feedback control: performance and robustness. IET Control Theory \& Applications, 2(7):595-605, 2008.

[25] M. Rotkowitz and S. Lall. A characterization of convex problems in decentralized control. IEEE Transactions on Automatic Control, 51(2):274-286, 2006.

[26] L. Schenato, B. Sinopoli, M. Franceschetti, K. Poolla, and S.S. Sastry. Foundations of control and estimation over lossy networks. Proceedings of the IEEE, 95(1):163 - 187, 2007.

[27] M.M. Seron, J.H. Braslavsky, and G.C. Goodwin. Fundamental Limitations in Filtering and Control. Springer, 1997.

[28] E.I. Silva. A Unified Framework for the Analysis and Design of Networked Control Systems. PhD thesis, School of Electrical Eng. and Comp. Sci., The University of Newcastle, Australia, 2009.

[29] E.I. Silva, G.C. Goodwin, and D.E. Quevedo. On the design of control systems over unreliable channels. In Proceedings of the European Control Conference, 2009.

[30] E.I. Silva, G.C. Goodwin, D.E. Quevedo, and M.S. Derpich. Optimal noise shaping for networked control systems. In Proceedings of the European Control Conference, 2007.

[31] E.I. Silva, D.A. Oyarzún, and M.E. Salgado. On structurally constrained $\mathcal{H}_{2}$ performance bounds for stable MIMO plant models. IET Control Theory \& Applications, 1(4):1033-1045, 2007.

[32] E.I. Silva, D.E. Quevedo, and G.C. Goodwin. Optimal controller design for networked control systems. In Proceedings of the 17th IFAC World Congress, 2008.

[33] D. Sourlas and V. Manousiousthakis. Best achievable decentralized performance. IEEE Transactions on Automatic Control, 40(11):1858-1871, 1995.

[34] O. Toker, J. Chen, and L. Qiu. Tracking performance limitations in LTI multivariable discrete-time systems. IEEE Transactions on Circuits and Systems I, 49(5):657-670, 2002.

[35] M. Vidyasagar. Control Systems Synthesis: A Factorization Approach. MIT Press, 1985.

[36] K. Zhou, J.C. Doyle, and K. Glover. Robust and optimal control. Prentice Hall, 1996. 